\title{
Apuntes vinculados a la importancia de la implementación del Compliance penal
}

Joaquín Missiego del Solar

Abogado por la Universidad de Lima.

Especialización en Derecho Penal por la Universidad de Salamanca. Profesor de Derecho Penal y Derecho Procesal Penal de la Universidad de Lima. Miembro del Consejo Consultivo de ADVOCATUS.

SUMARIO:

I. Antecedentes.

II. Consecuencias jurídicas de la comisión de un delito aplicables a la Persona Jurídica.

III. Sobre el Compliance.

IV. Sobre la legislación actual.

V. Conclusiones.

* El artículo fue recibido con fecha 25-05-19. 


\section{RESUMEN:}

De un tiempo a estar parte el término "Compliance" se ha incorporado fuertemente en el vocabulario jurídico, habiendo adquirido una gran importancia que no puede pasar de ninguna forma inadvertida hoy día, principalmente en el mundo empresarial. El no darle importancia traerá consigo una serie de contingencias, entre las cuales aparecen las de naturaleza penal, las mismas que serán objeto del presente artículo.

Resulta de vital importancia para las personas jurídicas, que establezcan políticas al interior de las mismas que les permitan desarrollar sus actividades en fiel cumplimiento con la normatividad vigente.

Palabras clave: naturaleza penal, norma, banco, riesgo, empresa.

\footnotetext{
ABSTRACT:

From a time to be part the term "Compliance" has been strongly incorporated into the legal vocabulary, having acquired great importance that cannot pass in any way unnoticed nowadays, mainly in the business world. Not giving it importance will bring with it a series of contingencies, among which appear those of criminal nature, which will be the subject of this article.

It is importance for legal entities to establish policies within them that allow them to develop their activities in full Compliance with current regulations.

Keywords: criminal nature, rule, bank, risk, company.
}

\section{INTRODUCCIÓN}

Uno de los principales antecedentes que trae consigo la implementación del Compliance en la actualidad en la mayoría de países, se encuentra en el año 2005. En el mes de abril de dicho año, el Comité de Supervisión Bancaria de Basilea —en adelante, "el Comité"—, publicó un documento titulado "Compliance and the Compliance Function in Banks", en el cual se establecen principios de alto nivel sobre la gestión de los riesgos de cumplimiento en los bancos. Posteriormente, como seguimiento a la publicación del documento, el Comité solicitó al grupo de trabajo de contabilidad que evaluara el grado de implementación de los principios de cumplimiento, así como que revisara los incidentes y desafíos recientes relacionados con el cumplimiento que enfrenta la industria.

El Compliance es un término que fue acuñado por el comité de Supervisión Bancaria de Basilea en 2005 con la siguiente definición:

“El Compliance es riesgo de que una organi- zación pueda sufrir sanciones, multas, pérdidas financieras o pérdida de su reputación como resultado de incumplimientos de las leyes, regulaciones, normas de autorregulación o códigos de conducta que se apliquen a su actividad".

Recordemos que cuatro años antes en el 2001, el caso Enron había generado una gran crisis y preocupación en el sistema financiero norteamericano, la cual se había irradiado más allá de sus fronteras. Al respecto, el abogado español, Dr. Miguel Linares Poleiro señala en un artículo publicado en la Guía Financiera Empresarial que:

"el Compliance nace en el mundo empresarial anglosajón, en concreto en Estados Unidos cuando, tras sonados casos de fraude y corrupción como el de Enron, WorldCom o Parmalat, se empezó a demandar públicamente un incremento en la regulación de las compañías, incluyendo la obligación de establecer controles efectivos que evidenciaran que las normas efectivamente se estaban cumpliendo"2.

1. Disponible en: <https://www.lopdencastellon.com/que-es-el-compliance-o-el-cumplimiento-normativo/>.

2. Disponible en: <https://www.guiafinem.com/la-funcion-de-compliance-garantiza-el-cumplimiento-de-la-nor$\underline{\text { mativa/>. }}$ 
Concluyendo en que la función del Compliance garantiza el cumplimiento de la normatividad.

En lo que refiere a nuestro país, la importancia e interés sobre el Compliance se viene acrecentando fuertemente en los últimos años; es así, que diversos medios de comunicación comenzaron a dedicar con mayor frecuencia espacios dedicados al tema. Si bien se ha publicado mucho sobre el tema, rescatamos de la prensa los aparecidos en algunos diarios, como es el caso de Gestión por ejemplo, donde se entrevistaban entendidos en la materia y refiriéndose al Compliance, o cumplimiento normativo, se señalaba que "consiste en establecer las políticas y procedimientos adecuados y suficientes para garantizar que una empresa, incluidos sus directivos, empleados y agentes vinculados, cumple con el marco normativo aplicable $e^{13}$.

Prueba del creciente interés sobre el tema, es el artículo que apareció en el mes de abril del último en Semana Económica, donde se indicaba:

"la necesidad de las grandes empresas por asesorías vinculadas al Compliance aumentó en el 2018. Así, la facturación de los estudios de abogados especializados en el rubro fue la que más aumentó el año pasado (...) El 2018 fue un año de cambios constantes. Nuevas tendencias regulatorias y el inicio de reformas importantes, como la tributaria y laboral, habrían afectado a las empresas en todos los sectores. A ello se suma el impulso del gobierno por la lucha contra la corrupción y la entrada en vigencia de la ley de responsabilidad de personas jurídicas, que aumentaron la demanda por los servicios relacionados a la implementación de programas de Compliance en las empresas' ${ }^{\prime 4}$.

En lo que refiere al aspecto legal, en el mes de abril del 2016 se promulgó la Ley 30424, pos- teriormente modificada por el Decreto Legislativo 1352. El objetivo de las normas es que las personas jurídicas implementen un programa de cumplimiento con el fin de disminuir o evitar su participación en delitos de cohecho activo transnacional, cohecho activo genérico, cohecho activo específico, lavado de activos y financiación del terrorismo.

\section{CONSECUENCIAS JURÍDICAS DE LA COMISIÓN DE UN DELITO APLICABLES A LA PERSONA JURÍDICA}

Un delito es una conducta humana voluntaria que se encuentra prohibida por la norma penal, la misma que no encuentra una causa de justificación - legítima defensa, estado de necesidad u obrar por disposición de la ley y, es cometida por un sujeto capaz o imputable-.

En ese contexto, se entiende que las personas naturales son las que comenten los delitos, pero debe tenerse presente que las consecuencias jurídicas producto de la comisión de un delito pueden afectar directamente a las personas jurídicas.

La consecuencia lógica de la comisión de un ilícito penal es la sanción al autor, la cual se pronunciará luego que se realice un proceso penal. En este contexto es necesario tener presente entonces que, la principal consecuencia de la comisión de un delito es la pena; sin embargo, también existen consecuencias de naturaleza civil y administrativa que surgen a raíz de la comisión de un delito, las cuales se conocen como consecuencias accesorias.

Todo ello no es un tema nuevo ni que se ha introducido en nuestra legislación a través de la importancia que ha venido dándole los órganos de dirección de las personas jurídicas al Compliance.

3. Disponible en: <https://gestion.pe/economia/empresas/compliance-empresas-peruanas-realizan-intenso-gestion-riesgos-237167>.

4. Disponible en: <https://semanaeconomica.com/article/legal-y-politica/marco-legal/340021-los-estudios-especializados-en-compliance-asesoraron-a-las-empresas-mas-grandes-en-el-2018/>. 
Como sabemos la responsabilidad civil proveniente del delito tiene su base en la llamada responsabilidad extracontractual que aparece en el Código Civil —artículo 1969- donde se señala que quien causa un daño a otro está obligado a indemnizarlo. La comisión de un delito causa un daño y, es ahí donde encontramos la razón y la lógica de aplicar medidas de naturaleza civil en el ámbito de un proceso penal. Por ende, si los autores del delito se valen de la persona jurídica para la comisión del hecho delictivo, este acto genera consecuencias en ella.

Es así; que el Código Penal cuando se refiere a la responsabilidad civil proveniente del delito y a las sanciones administrativas aplicables a las personas jurídicas sostiene en el artículo 95 que la responsabilidad civil es solidaria: "La reparación civil es solidaria entre los responsables del hecho punible y los terceros civilmente obligados".

En lo que respecta a las medidas aplicables a las personas jurídicas, en el artículo 105 prescribe que:

"Si el hecho punible fuere cometido en ejercicio de la actividad de cualquier persona jurídica o utilizando su organización para favorecerlo o encubrirlo, el Juez deberá aplicar todas o algunas de las medidas siguientes:

1. Clausura de sus locales o establecimientos, con carácter temporal o definitivo. La clausura temporal no excederá de cinco años.

2. Disolución y liquidación de la sociedad, asociación, fundación, cooperativa o comité.

3. Suspensión de las actividades de la sociedad, asociación, fundación, cooperativa o comité por un plazo no mayor de dos años.

4. Prohibición a la sociedad, fundación, asociación, cooperativa o comité de realizar en el futuro actividades, de la clase de aquellas en cuyo ejercicio se haya cometido, favorecido o encubierto el delito.

La prohibición podrá tener carácter temporal o definitivo. La prohibición temporal no será mayor de cinco años. Cuando alguna de estas medidas fuera aplicada, el Juez ordenará a la autoridad competente que disponga la intervención de la persona jurídica para salvaguardar los derechos de los trabajadores y de los acreedores de la persona jurídica hasta por un período de dos años. El cambio de la razón social, la personería jurídica o la reorganización societaria, no impedirá la aplicación de estas medidas".

El legislador también tuvo en cuento un grupo de criterios que sirviesen de marco para la determinación de las consecuencias aplicables a las personas jurídicas, las cuales son, según el caso:

"1. Prevenir la continuidad de la utilización de la persona jurídica en actividades delictivas.

2. La modalidad y la motivación de la utilización de la persona jurídica en el hecho punible.

3. La gravedad del hecho punible realizado.

4. La extensión del daño o peligro causado.

5. El beneficio económico obtenido con el delito.

6. La reparación espontánea de las consecuencias dañosas del hecho punible.

7. La finalidad real de la organización, actividades, recursos o establecimientos de la persona jurídica.

La disolución de la persona jurídica se aplica siempre que resulte evidente que ella fue constituida y operó habitualmente para favorecer, facilitar o encubrir actividades delictivas".

A estas alturas podemos llegar a una primera conclusión, el Compliance no es un mecanismo que haya surgido con la finalidad de sancionar, sino todo lo contrario; lo que se busca con el Compliance es aplicar un modelo de prevención que evite las sanciones a las que pudiera exponerse la empresa.

\section{SOBRE EL COMPLIANCE}

En principio debemos tener en claro que el concepto Compliance debe girar sobre las bases de 
la ética y el buen gobierno corporativo. Se trata de una "forma de vida empresarial" donde prime y destaque la actuación de cada uno de los miembros de una persona jurídica, respetando las normas y los procedimientos legales para obtener los mejores resultados.

La frase "el fin justifica los medios" no es de ninguna manera bien recibida, ni va de la mano con el Compliance y las buenas prácticas corporativas. Hoy en día las empresas deben tener en claro que deben evitar involucrarse en actos vinculados a la comisión de delitos, principalmente a temas relacionados con la corrupción; así como también generar los procedimientos adecuados que le permitan desempeñarse respetando los parámetros normativos vigentes.

Viene a la memoria las llamadas normas ISO, cuya finalidad es implementar un conjunto de normas vinculadas a la eficiente gestión, en este caso de la persona jurídica. Podríamos relacionarlo como una referencia oportuna para entender el Compliance.

Muchas veces a raíz de verse involucradas en actos delictivos, malas prácticas o simplemente por falta de cuidado, empresas importantes han visto disminuir su marcha regular, a consecuencia de manchar su reputación justamente por no tener en claro cómo llevar a cabo las llamadas buenas prácticas corporativas.

Una importante institución llamada World Compliance Association, cuya finalidad según señalan es la promoción, reconocimiento y evaluación de las actividades de cumplimiento en las organizaciones, independientemente de su forma jurídica, así como el desarrollo de herramientas y procesos para una correcta protección frente a determinados delitos/infracciones cometidas por sus empleados, colaboradores o cualquier otra persona relacionada con ella, define el Compliance señalando que:

"El Corporate Compliance es un conjunto de procedimientos y buenas prácticas adoptados por las organizaciones para identificar y clasificar los riesgos operativos y legales a los que se enfrentan y establecer mecanismos internos de prevención, gestión, control y reacción frente a los mismos.

El Compliance en muchos casos, y a tener de la evolución del marco legal a nivel mundial y la clara tendencia en este sentido, ha dejado de ser una opción voluntaria para muchas organizaciones y ha pasado a ser un requisito a integrar dentro de su estrategia y estructuras internas a fin de dar cumplimientos a los preceptos legales o bien poder protegerse ante situaciones de riesgo que pondrían en serios problemas la estabilidad y continuidad de la actividad de la organización.

Por ello, y por los recientes escándalos societarios y el innegable incremento de la sensibilidad social respecto de la ética de los negocios, un mayor número de organizaciones públicas y privadas internalizan estándares éticos y legales como protocolos de buen gobierno de obligado cumplimiento.

Los riesgos a prevenir son aquellos que conllevan consecuencias como el daño reputacional, la imposición de importantes multas y sanciones, las pérdidas de negocio por contratos no ejecutables o la exclusión de licitaciones o subvenciones públicas, entre otras"s.

De acuerdo a lo señalado anteriormente, las personas jurídicas pueden ser consideradas como tercero civil en el ámbito de un proceso penal y a raíz de ello, ser objeto de sanciones de naturaleza civil y administrativa provenientes de la comisión de un delito. En ese contexto, durante el desarrollo de una investigación de naturaleza penal, una de las clásicas preguntas que le hacen al representante de la persona jurídica es: ¿qué hicieron ustedes para evitar que esto sucediera?; y, la respuesta debe venir acompañada de todos los elementos de prue-

5. Disponible en: <http://www.worldcomplianceassociation.com/que-es-compliance.php $>$. 
ba que permitan acreditar que la persona jurídica "actuó diligentemente".

Si queremos simplificar el tema, los elementos básicos vinculados al Compliance no están solo en función a tener a la mano elementos de prueba que permitan acreditar la actuación de la empresa frente al delito, sino a que los procedimientos al interior de la empresa traigan consigo valores éticos que le permitan tomar decisiones y desempeñarse de la manera más eficiente.

El Compliance también permite a los empresarios evitar una serie de multas o cualquier otro tipo de sanción; en efecto, las buenas prácticas corporativas, el respeto a la normatividad, les evita o disminuye contingencias económicas. Resulta mejor invertir - no gastar- en un sistema de prevención que tener que estar pagando por las infracciones cometidas, las cuales como se ha señalado no solo conllevan aspectos de naturaleza civil, sino también administrativos que pueden derivar en la suspensión de actividades, retiro de licencia $u$ otras que pongan en peligro el normal desarrollo de la persona jurídica.

El portal Legis nos presenta al Profesor Dr. Santiago García Echevarría, de la Universidad de Alcalá, señalando que en su trabajo Conferencias y Trabajos de Investigación del Instituto de Dirección y Organización de Empresas /Núm. 342 - Problemas de Organización Empresarial de Los Programas De Compliance, definió que el Compliance:

"constituye un nuevo elemento legal que enmarca tanto la configuración y desarrollo de la dirección de la empresa y sus procesos como la responsabilidad societaria, corporativa, de las organizaciones. El legislador impone un marco legal de normas de com- pliance y las propias corporaciones han desarrollado normas internas, con mayor o menor fortuna, para el desarrollo de las responsabilidades de directivos y corporaciones" ${ }^{\prime \prime}$.

Finalmente cabe resaltar lo señalado por la Dra. Elizabeth Peralta Quipe cuando sostiene que:

"(...) es importante mencionar que el espiritu de la normativa coloquialmente conocida como "Compliance Penal" busca que las personas jurídicas integren en su ADN prácticas que fomenten la prevención de comisión de delitos, esto puede apreciarse así de las nuevas disposiciones del Reglamento, que impulsan la integración del Modelo de Prevención en el día a día de las personas jurídicas"'.

\section{SOBRE LA LEGISLACIÓN ACTUAL}

Como antecedente podemos mencionar que en abril del 2016, se promulgó la Ley 30424 la cual regulaba la Responsabilidad Administrativa de las Personas Jurídicas, posteriormente en enero del presente año se modifica mediante el Decreto Legislativo 1352, el cual amplía el ámbito de la Responsabilidad Administrativa de las Personas Jurídicas y por la Ley 30835 , se reguló la Responsabilidad Administrativa de las Personas Jurídicas por el delito de cohecho activo transnacional, que estableció que las personas jurídicas pueden ser declaradas responsables administrativamente de la comisión de los delitos de cohecho transnacional, cohecho activo genérico, cohecho activo especifico, colusión simple y agravada, tráfico de influencias, lavado de activos y financiamiento del terrorismo.

Es importante resaltar que el artículo 17 de la mencionada Ley, presenta un supuesto de exención de responsabilidad administrativa de la persona jurídica por la comisión de los delitos de cohecho transnacional, cohecho activo ge-

6. Disponible en: <https://legis.pe/recomendaciones-implementar-plan-compliance-prevencion-delitos-empre$\underline{\mathrm{sa} />}$.

7. Disponible en: <https://www.tytl.com.pe/comentarios-acerca-del-reglamento-de-la-ley-30424-ley-que-regula-la-responsabilidad-administrativa-de-las-personas-juridicas/>. 
nérico, cohecho activo especifico, colusión simple y agravada, tráfico de influencias, lavado de activos y financiamiento del terrorismo, cuando hubiera adoptado e implementado en su organización y con anterioridad a la comisión del delito, un modelo de prevención adecuado a su naturaleza, riesgos, necesidades y características, consistente en medidas de vigilancia y control idóneas para prevenir los delitos antes mencionados o para reducir significativamente el riesgo de su comisión.

Al respecto, reiteramos la pregunta hecha anteriormente: ¿qué hicieron para evitar que esto sucediera? La respuesta se encuentra en la implementación del modelo de prevención adecuado señalado en la norma; sin embargo, a efectos de ordenar los criterios para implementar el modelo, consideramos pertinente la intención de reglamentar la Ley 30424 mediante el Decreto Supremo N 002-2019-JUS.

Al respecto, en un interesante artículo publicado en Legis escrito por los doctores Caridad Molina y Raúl Pérez, se señala:

“(...) para que el Modelo de Prevención actúe como eximente o atenuante de responsabilidad penal, éste debe ser declarado adecuado por parte de la SMV a través de un informe técnico solicitado por el Fiscal. El Reglamento ha definido cuales son los parámetros que podrán ser evaluados por la SMV para determinar si un Modelo de Prevención de Delitos es adecuado, o no. Estos parámetros a modo de resumen son los siguientes: (i) Análisis y corrección de los riesgos inherentes y residuales; (ii) Compromiso y liderazgo de los órganos de gobierno; (iii) Autonomía y recursos; (iv) Políticas y Procedimientos; (v) Evaluación de Riesgos; (vi) Formación y Comunicaciones; (vii) Informes Confidenciales e Investigación; (viii) Incentivos y medidas disciplinarias; (ix) Mejora Continua, Pruebas Periódicas y Revi- sión; (x) Gestión de Terceros; y (xi) Fusiones y Adquisiciones".

Resulta importante tener en cuenta las referencias a las cuales ley hace mención. Fija los lineamientos a seguir por las personas jurídicas con el fin de evitar sanciones desde una visión moderna, en función a la prevención y no a la represión.

La prevención trae consigo políticas vinculadas a un comportamiento y trabajo probo, leal y legal, con lo cual finalmente quien se beneficiaría no es solo la persona jurídica, sino la sociedad en general en la medida que vería disminuida la incidencia de casos de corrupción empresarial, que lamentablemente se ha venido repitiendo con frecuencia en nuestra sociedad.

\section{CONCLUSIONES}

a) Sin lugar a dudas el Compliance hoy en día, es un instrumento necesario e imprescindible en toda persona jurídica que se considere seria y diligente en su accionar.

b) Los procedimientos necesarios que permiten el avance y crecimiento empresarial de la mano de las normas vigentes, posibilitan un normal desarrollo de las actividades de las personas jurídicas, evitando contingencias que deriven en obstáculos - sanciones- para su marcha regular.

c) El incumplimiento de las reglas vinculadas al Compliance, traerá consigo una situación de grave riesgo en la persona jurídica que podrá traer generar consecuencias negativas en el desarrollo de la misma.

d) El Compliance deberá ser adoptado como una forma adecuada de comportamiento de la persona jurídica y sus miembros, en busca de la transparencia y ética empresarial.

8. Disponible en: <https://legis.pe/comentarios-d-s-002-2019-jus-reglamento-ley-30414-regula-responsabilidad-administrativa-penal-personas-juridicas/>. 